\title{
Beneath the Himalayas - Exploring Design for Cultural Evenness with Nepalese Children
}

\author{
Dev Raj Lamichhane \\ University of Central Lancashire \\ Preston, UK \\ DRLamichhane@UCLan.ac.uk
}

\author{
Janet C Read \\ University of Central Lancashire \\ Preston, UK \\ JCRead@Uclan.ac.uk
}

\author{
Dan Fitton \\ University of Central Lancashire \\ Preston, UK \\ DBFitton@Uclan.ac.uk
}

\begin{abstract}
This paper describes a participatory study with children from a Nepalese village in the Himalayan Mountains. The aim of this work is to derive requirements for a mobile app that will allow children in developing rural communities (in countries such as Nepal) to share stories, without the use of English, with children in more developed urban communities (in counties such as the UK).The children, aged 9-11, were asked to draw their local environments as design inputs to a characterbased interface for the mobile application. This paper describes how the study was conducted, gathers together the results and then reflects on both the usefulness of the drawn images for design and also the usefulness of the drawing-based design method. The work is novel in its context of place and use and the work draws useful findings for others seeking to do such work in faraway places.
\end{abstract}

Cultural Evenness, Cross Cultural Communication, Child Computer Interaction.

\section{INTRODUCTION}

The world is a place that is full of tensions and conflicts. Technology is able to intersect in positive ways to bring communities and people together where they can learn from one another and where tolerance of differences can be fostered. Children are typically much more open to ideas around difference and diversity than older people and as they learn about the world there is a great oportunity to develop technologies, systems and solutions that assist in this endeavour.

Technology innovations that speak to inclusion and tolerance are typically designed and developed in the developed world. This approach can lead to products being designed that do not take account of local contexts but also result in products that are biased considerably in favour of Western ideals and Western content. (Brewer et al., 2005).

One response to this is to consider interfaces in terms of their cultural appropriateness. Work has been done in this area especially in terms of HCl4D; one example is a study done in Nairobi, Kenya that showed how the constraints of security, cost, bandwidth and responsiveness in that environment impacted on ICT design (Wyche et al., 2010).

Cross-cultural communication is difficult when the two cultures do not share a commmon language $(\mathrm{Ho}$ et al., 2009). Classic solutions to this aim to remove language barriers by either using International English and translation technologies or by using image based interfaces. Whilst these fixes go some way towards making interfaces useful across cultures, they only account for cultural variety on a surface level and do little to mitigate away from a deep seated Western paradigm of interface design. Language translation is especially difficult when the inteneded users of the technology are children. Children will typically be much less proficient with a second language and the errors and mistakes they make in their own languages will make any automatic translation more error prone (Read et al., 2002).

Our work in this paper represents the commencement of a journey towards 'cultural evenness' as a design construct for collaborative technologies for children. We position cultural evenness as being an ideal to strive for as opposed to a design requirment. We illustrate our thinking in the examination of a set of design cameos gathered from children in a remote village in the Nepalese Himalayas. Our paper begins with an overview of the literature, leading on to our definition of cultural evenness before reporting on the design study.

\section{RELATED WORK}

\subsection{Communication in Different Cultures}

The most pressing need for a cross cultural interface is for it to be understood by both parties at the initial user interface layer which we refer to as presentation. Classically this is where the written and spoken language of the users is the barrier or the entry point to any meaning that is imbued in the interface. Early $\mathrm{HCl}$ work pressed for the use of a built-in translation of text on user interfaces (often denoted with a flag of the different countries) and / or images to make access to the interface intuitive. The use of some icons is considered almost universal especially with the wide uptake of computer 
systems. In the same way that icons are universal, so, to a lesser extent is the use of the Latin character set for communication, and consequently the use of English (albeit International or often American) as the default language of systems.

Pictoral representation can also be used to convey simple sentences in language barrier environment which is facilitated with the help of PicNet illustrated dictionary and automatic machine translation system (Mihalcea \& Leong,2009).

\subsection{PenPal Interfaces for Children}

Having a 'pen pal' at school was a fairly common activity for children in the 50 s and 60 s and was encouraged to improve cross cultural understanding and to encourage children to write and read in 'foreign' languages. Shulman et al., (1994) reported on how many school-initiated pen pal relationships continued into adolescence and how many of these relationships were considered safe, close and open.

Choosing what to write about, especially at the start of a pen pal relationship, is quite challenging. In 2007, Barksdale et al., studied pen pal letter exchanges between 90 students from America and 85 students from Malawi. They found that the students were typically talking about their daily lives, their cultures and languages. A similar study was done with children from Zambia and America where children shared stories about their life-styles, culture, weather and country (Shandomo, 2012).

In some cases, pen pal relationships were intended to promote the English language as a dominant expressive form. A study in Taiwan with $4410^{\text {th }}$ grade students found that a technology-enhanced pen pal helped them learn and improve their English letter writing skills (Yang \& Chen, 2006). Most technology products fail to include cross cultural equity and language less communication (Piernot et. Al, 1994).

\section{CULTURAL EVENNESS AS A DESIGN CONSTRUCT}

$\mathrm{HCl}$ has a series of general and implicit design constructs. These have evolved over time with early ones including efficiency and effectiveness (central to usability) and with new constructs being added as users' different needs are identified; with accessibility being one such construct and collaboration being another.

We define in this paper the construct of 'cultural evenness' to be an aim for design, especially for children's technologies. When children meet a technological system, the values which are imbued in it have a greater effect on the child than would be the case in an adult interface. This is because for the child, values and opinions are still very much being formed and the child is often very open to suggestion. Values that software brings include cultural values such as reward structures, values around how communities operate (together or in opposition), values around rule following and values around winning and losing. These culturally situated values can be subtly apparent in technologies that may then have a veneer of cultural adaptation put onto them so as to appear culturally neutral.

We define cultural evenness as an approach to design that seeks to not favour one culture over another and that seeks to not promote a bias towards one particular cultural view.

We see cultural levelling (being the process towards evenness) as beginning with the presentation layer that being what is seen on the interface of a product but then going below this to extend to how a system operates in terms of its rewards, its philosophy and its weltanschauung. This is a deep and complex problem space and one that will take numerous research studies. In this particular study we focus on that top layer; presentation - and ask the question how can we be informed by children on how to design for cultural evenness?

\section{RESEARCH DESIGN}

This paper describes a fieldwork study aimed at understanding the place of cultural evenness. It was carried out in a small school in a village in Nepal. The fieldwork was done by the first named author on this paper and the other authors contributed to the analysis and paper writing.

\subsection{Participants:}

The child participants in the study were 35 students chosen by the school Head Teacher. They were aged 9-11. They each had the study clearly explained to them. Each child, and his / her parents, consented to take part.

\subsection{Procedure:}

The study had to be done in the language of the children. In this way, the research was conducted by a Nepalese speaker who, even then, had some barriers with the children who also spoke a local dialect. The children were asked to draw something about themselves that they would write in a letter to another child to introduce himself or herself. Each child was given a piece of paper and coloured pencils. They were free to draw whatever they wanted to and no instruction was given as to what to draw. There was no time limit imposed because that might put constraints on how much or on what they wanted to express. The children passed their drawings to the researcher once they had finished. 


\subsection{Results:}

35 drawings were collected from the children - these contained 335 different elements which represented 75 items. (as an example, 25 children each drew the school - this contributes to the total number of elements - with the school being one item).

Of the 75 items, only three could be described as 'not things'. The remaining items were all tangible things that clearly existed within view of the child or represented something that was special to them. The items drawn the most are shown in table 1.

Table 1- The frequencies of common items

\begin{tabular}{|l|l|}
\hline \multicolumn{1}{|c|}{ item } & $\begin{array}{c}\text { Number of children } \\
\text { including it }\end{array}$ \\
\hline Home & 29 \\
\hline School & 25 \\
\hline Temple & 18 \\
\hline Flowers & 15 \\
\hline Mountain & 13 \\
\hline Path & 13 \\
\hline Duck & 12 \\
\hline Bird & 12 \\
\hline Sun & 12 \\
\hline Trees & 11 \\
\hline
\end{tabular}

Themes

Following guidelines from Bogdan and Biklen (1988), using open thematic analysis, we classified the items on the drawings into 5 categories;

Landscape: Children drew features of their landscape - given that this was Nepal and in the foothills of the Himalayas, mountains featured on many of the drawings - and these were often punctuated with the sun or with snow or both. Other landscape items included trees and rivers.

Community: Items that showed how the community had structured itself were also present. These included the school, the temple, houses, bridges etc. Almost all the children had most of these on their pictures which could be taken as them understanding the importance of these structures for them. The pictures of the school were especially well detailed with many children showing all the rooms, all the stairways, all the paths etc.

Family: Many, but not all, children expressed something about the family and their possessions by drawing their individual farming fields, their own homes, their animals and their own gardens.

Identity: The children were delighted to share things that would identify themselves or the whole country. For example. the national flag, national bird, national flower, Mt. Everest etc. Associated with this, they also were keen to draw the new things that had just come to their village; notably the new road, the new school tap and the new hospital.
Themselves: Favourite foods were included in many of the pictures, and favourite things including birds and flowers and butterflies. It was noted that there were no toys included and personal possessions appeared very limited. Several children drew family members.

\section{Observations}

The children mainly drew 'things' but there were some non-tangible things represented in the drawings. One drew love hearts, and another wrote welcoming words around the Nepalese flag. Clearly drawing non-tangible things is much harder than drawing things.

It was also quite difficult in some cases to see what the drawings were of, some things, like the school and the temple were very obvious, but other things, like lentils and rice and mangos and oranges had to be labelled. In the main the children seemed to understand what 'needed' labelling and included labels for such things - viz. the school was seldom labelled but fruits often were.

During the analysis a small number of assumptions were made about the implied meaning in the drawings. For example, that the lots of flowers together represented a garden and that lots of trees together represented a forest. These were discussed and agreed on between all the coders.

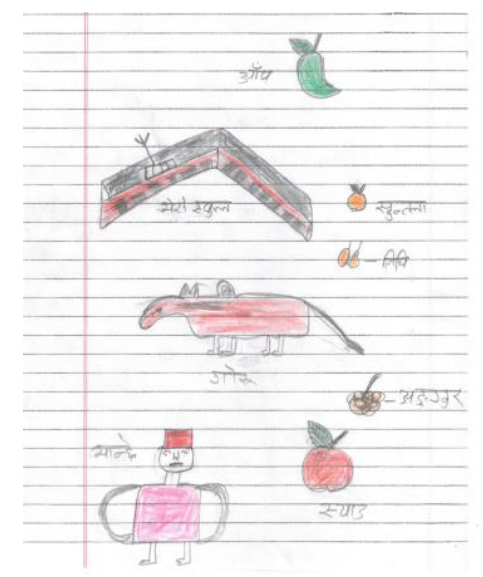

Figure 1: List of different items - showing the school, some fruit, a cow and a person

\section{Way of representation}

Children represented their environments through drawings in one of two ways. Either they drew the things all over the page - as a list (see Fig 1) or they drew items into one complete picture consisting of everything that they wanted to convey in a connected and relational structure (see Fig 2). 


\section{DISCUSSION}

\subsection{Informing Design}

In terms of the design of the new app, the drawings told us several important things about communication of stories without the use of words. Firstly, they perhaps gave us a sense of the initial

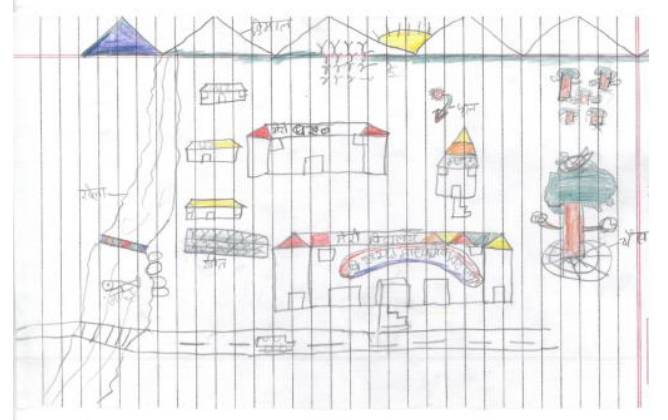

Figure 2: Everything in one picture - this includes the mountains with the sunshine on them, the school in the centre, houses, trees, the new tap, a forest, the new road, the river and a field. The tree has an area underneath it where children would sit around and spend a nice time in the sunshine.

dialogue of a pen pal conversation where the writer is saying what they have. The writer explains their landscape and in this case these pictures work quite well. It would also seem to be possible to simply have a selection of photos of the landscape as a way to show what the landscape holds but there would be things there that an observer from another place would not be able to label and this would be a problem.

In the analysis of the pictures, the local researcher from Nepal was able to 'translate' some of the pictures for the UK researchers. This may suggest that our app might need a mediator to add some translation to the pictures.

What the pictures did not tell us was about 'what was happening' which would seem to be the next stage that would be needed followed by 'what was being experienced/ felt'.

\subsection{Effectiveness of the Method}

The method was effective for capturing themes that might be important to the children in the way they would describe their spaces. The five themes that emerged - landscape, community, family, identity and self were populated in different densities with very little being about the individual child and much more being about the spaces around the child. This exposed some of the cultural position of this group where the land and the community are very important. The method allowed us to describe these children's contexts - as being often outdoors, being family and community centred, proud of their community, proud of their natural landscapes and at one with the animals and birds and flowers in their locality.

The central position of the school in many of the pictures was also useful in seeing how they 'saw' their community. The school was, for the children, their focal point and many had put a great deal of effort into drawing this with great accuracy so that we could be impressed by its scale and design.

\subsection{Towards Cultural Evenness}

In terms of what we could learn towards our ideal for Cultural Evenness, this study exposed three key things;

(i) Capturing language with pictures will be hard. For a first introduction, images of landscapes may be helpful but there may need to be some explanation of content that is obvious in one context but unknown in another

(ii) Children are aware of what can and cannot be decoded by others - in this study they labelled things that were possibly ambiguous - in our app we will need to facilitate a way for children to mark ambiguous things

(iii) If children are able to select a set of things to include in a 'conversation', with these selections we may be able to make some assumptions about some of the community ideals of that culture that could inform other aspects below the presentation layer.

For cultural evenness these observations suggest the following design ideas - possibilities to upload photos and tag things as 'hard to guess what these are', that we may need an adult to look over some of the content to effect a translation of sorts, and that we may be able to use a form of crowdsourcing of content to set some of the cultural parameters for a set of users,

\section{CONCLUSION}

In this work we have made the case for, and described, the idea of cultural evenness as a design characteristic for children's cross cultural conversational interfaces. We have identified, for one culture, how drawings can convey their 'as is' and have realised three challenges for the implementation of a language free interface for a children's pen pal application.

The work is now being followed with the same children writing letters in order that we can see what is common to both media and to see if we find out more about what the children do when they use words. We are also gathering pictures from children in the UK so we can see how these differ. 


\section{REFERENCES}

Barksdale, M. A., Watson, C., \& Park, E. S. (2007). Pen Pal Letter Exchanges: Taking First Steps Toward Developing Cultural Understandings. The Reading Teacher, 61, 58-68.

Bogdan, R.C., \$ Biklen, S. K., (1988). Qualitative research for education: An introduction to theory and methods ( $3^{\text {rd }}$ ed.).Boston: Allyn \& Bacon

Brewer, E.,Demmer, M., Du, B., Ho, M., Kam, M., Nedevschi, S., Pal, J., Patra, R., Suran, S \& Fall, K. (2005). The case for technology in developing regions. Computer, 38(6), 25-38.

Ho, M. R., Smyth, T. N., Kam, M. \& Dearden, A. (2009) Human Computer Interaction For Development: The Past, Present, and Future. Information technology and international development, 5(4).

Mahalcea, R., \& Leong, C.W. (2009) Toward communicating simple sentences using pictorial representations. Springer Science+Business Media B.V.

Piernot, P.P., Felciano, R. M., Stancel, R., Marsh, J., \& Yvon, M., (1995). Designing the Penpal: blending hardware and software in a userinterface for children. In Proceedings of the
SIGCHI Conference on Human Factors in Computing Systems (CHI'95), New York

Read, J. C., S. J. MacFarlane and C. Casey (2002). Oops! Silly me! Errors in a Handwriting Recognition-based Text entry Interface for Children. NordiChi 2002, Aarhus, Denmark, ACM Press

Shandomo, H. M. (2012). Getting to Knew You: Cross-Cultural Pen Pals Expand Children's World View. Childhood Education, 85(3), 154-159.

Shulman, S., Seiffge-Krenke, I., \& Dimitrovsky, L., (1994). The functions of pen pals for adolescents. Journal of Psychology, 128, 89-100.

Wyche, S. P., Smyth, T. N., Chetty, M., Aoki, P. M., \& Grinter, R. E. (2010). Deliberate interaction: characterizing technology use in Nairobi, Kenya. Paper presented at Proceedings of the SIGCHI Conference on Human Factors in Computing Systems (CHI'10). ACM, New York.

Yang, S. C., Chen, Y., (2006). Techonolgyenhanced language learning: A case study, Science Direct, Computers in Human Behavior, 23, 860-879 